\title{
Low percolation concentration for zero thermoelectric power in $\mathbf{Y}_{1} \mathrm{Ba}_{2} \mathrm{Cu}_{3} \mathbf{O}_{7-x}$
}

\author{
S R JHA, Y S REDDY and R G SHARMA* \\ National Physical Laboratory, New Delhi 110012, India.
}

\begin{abstract}
Effect of oxygen deficiency on the thermoelectric power and the resistive transition is studied by changing the cooling rate of the sintered specimens of $\mathrm{R}_{1} \mathrm{Ba}_{2} \mathrm{Cu}_{3} \mathrm{O}_{7-x}$ where $\mathrm{R}=\mathrm{Y}, \mathrm{Gd}$ and $\mathrm{Sm}$. Slow-cooled $\left(60^{\circ} \mathrm{C} / \mathrm{h}\right)$ specimens are superconducting and show positive TEP with a broad peak above $T_{c}$. It is a decreasing function of temperature at higher temperatures. Pair fluctuation above $T_{c}$ is thought to be responsible for this peak. Highly $\mathrm{O}_{2}$-deficient sample of $\mathrm{Y}_{123}$ has a finite resistivity at $T_{c}$ and yet shows a zero TEP. It is suggested that the percolation concentration of the superconducting phase for zero TEP should be much smaller than that required for the electrical conduction.
\end{abstract}

Keywords. Percolation threshold; thermoelectric power; oxide superconductors; Josephson coupling.

\section{Introduction}

The high $T_{c}$ oxide superconductors are characterized by several unique features such as the granular nature, a layered structure and the presence of a gaseous component - oxygen. These features lead to highly anomalous transport properties in their normal state and exhibit superconducting behaviour quite different from the conventional superconductors. Large anisotropies observed in resistivity, TEP, $H_{c 2}$ and $J_{c}$ in $a-b$ plane and along the $c$-axis (Dinger et al 1987; Hagen et al 1988; Martin et al 1988; Wang and Ong 1988) make these superconductors a class of their own. The granular nature of the oxide superconductor is the single biggest problem in realizing the large transport critical current $J_{c}$, in polycrystalline bulk material and therefore requires a thorough study of the percolation problem. These superconductors consist of isolated grains with good superconducting characteristics separated by regions which are weakly superconducting or could be even insulating (Taylor et al 1988). The conduction through the grains is governed by the strength of the intergrain coupling (weak links) which is usually assumed to be of Josephson type (Deutscher et al 1980). The grains are coupled when the Josephson coupling energy $\left(E_{j}\right)$ exceeds the thermal energy $\left(K_{B} T_{c}\right)$. As the temperature is lowered, more and more grains are coupled until at a temperature the coupling probability becomes equal to the percolation threshold. This temperature marks the transition temperature $T_{c}$ where an infinite cluster of coupled superconducting grains exist.

The oxygen stoichiometry is most crucial to the superconductivity in the $\mathrm{Y}_{1} \mathrm{Ba}_{2} \mathrm{Cu}_{3} \mathrm{O}_{7-x}$ class of $90 \mathrm{~K}$ superconductors (Jorgensen et al 1987). Oxygen deficiency in $Y_{123}$ material leads to gradual vacancies at the $\left(0, \frac{1}{2}, 0\right) O$-sites and occupancies at the vacant $\left(\frac{1}{2}, 0,0\right)$ sites resulting in a second-order transition at $X=0.5$ from an orthorhombic to a tetragonal phase which is not superconducting.

* For correspondence 
In most cases oxygen is less than 7 and the material is inhomogeneous consisting of orthorhombic and tetragonal phases. As mentioned earlier the resistivity will drop to zero at $T_{c}$ if the volume fraction of the superconducting phase is above the percolation threshold which for a metal-insulator (or a superconductor-metal) system is about $17 \mathrm{~V} \%$ (Zallen and Scher 1971).

This paper attempts to show that the thermoelectric power (TEP) of a system with a superconducting volume fraction (SVF) much smaller than the percolation threshold is zero at $T_{c}$ even though the resistivity is finite. The TEP is indeed a parameter much more sensitive to the homogeneity of these granular materials than the electric resistivity. The weak links do not affect the thermal transport to the extent they affect the electrical conduction. TEP does not depend on the sample geometry either and yields information on the nature of charge carriers and their interaction with phonons. Experimental data on the resistivity and the TEP of the well-annealed and $\mathrm{O}_{2}$-deficient $\mathrm{R}_{1} \mathrm{Ba}_{2} \mathrm{Cu}_{3} \mathrm{O}_{7-x}(\mathrm{R}=\mathrm{Y}, \mathrm{Sm}$ and $\mathrm{Gd})$ specimens are presented. Percolation in relation to the TEP behaviour is discussed.

\section{Experimental}

The powder samples of $\mathrm{Y}_{1} \mathrm{Ba}_{2} \mathrm{Cu}_{3} \mathrm{O}_{7-x}, \mathrm{Sm}_{1} \mathrm{Ba}_{2} \mathrm{Cu}_{3} \mathrm{O}_{7-x}$ and $\mathrm{Gd}_{1} \mathrm{Ba}_{2} \mathrm{Cu}_{3} \mathrm{O}_{7-x}$ were prepared by the usual solid-state ceramic technique as described earlier (Sharma et al 1988). Preformed powders were then pressed to form rectangular bars $(35 \times 4 \times 3 \mathrm{~mm})$ which were sintered at $920^{\circ} \mathrm{C}$ for $15 \mathrm{~h}$ under flowing oxygen. One set of specimens were cooled down slowly as usual $\left(60^{\circ} \mathrm{C} / \mathrm{h}\right)$ and another set quenched to $77 \mathrm{~K}$. Electrical resistivity of all the specimens was measured by the usual four-probe method in a variable temperature cryostat.

TEP was measured between 300 and $77 \mathrm{~K}$ by a method described earlier (Jha et al 1989a). In brief, the bar-shaped specimen was mounted in a vacuum can with the top end in good thermal contact with the lid of the can. A temperature gradient of $2-3 \mathrm{~K}$ was maintained using a heater wound at the bottom end of the specimen. $\Delta T$ was measured with a differential copper-constantan thermocouple. The thermoelectric voltage was measured with a Keithley nanovoltmeter. Room temperature XRD spectra were recorded between $2 \theta$ values of $20^{\circ}$ and $70^{\circ}$ on a Philips X-ray diffractometer. The lattice parameters were calculated from the well-resolved peaks $\operatorname{viz}(200),(020)$ and $(006)$ between $2 \theta=46$ and $48^{\circ}$ using slow scan at $1 / 8^{\circ}$ per minute.

The oxygen content in the specimens was determined with a gas chromatograph (model Perkin Elmer Sigma 2000) specially adapted for the purpose (Parashar et al 1988). The oxygen content in the quenched specimens was found to be $\leqslant 6.5$.

\section{Results and discussions}

\subsection{Slow-cooled specimens}

The resistive transitions and the TEP vs temperature plots of the three systems viz. $\mathrm{Y}_{1} \mathrm{Ba}_{2} \mathrm{Cu}_{3} \mathrm{O}_{7-x}, \mathrm{Gd}_{1} \mathrm{Ba}_{2} \mathrm{Cu}_{3} \mathrm{O}_{7-x}$ and $\mathrm{Sm}_{1} \mathrm{Ba}_{2} \mathrm{Cu}_{2} \mathrm{O}_{7-x}$ in their slow-cooled $\left(60^{\circ} \mathrm{C} / \mathrm{h}\right)$ and quenched $(77 \mathrm{~K})$ are shown in figures 1 to 3 . As evident from the plots the slow-cooled specimens of the three systems show superconducting transition with 


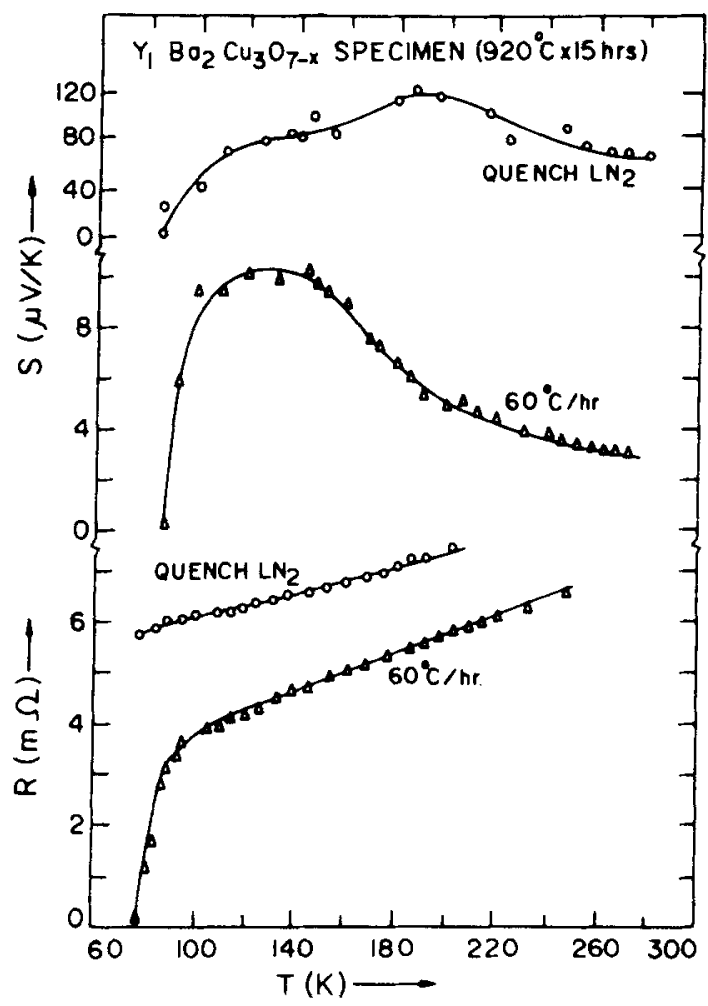

Figure 1. Measured thermoelectric power and electrical resistance, of the $\mathrm{Y}_{1} \mathrm{Ba}_{2} \mathrm{Cu}_{3} \mathrm{O}_{7-x}$ specimens. Note that the TEP of the quenched sample, though large, yet drops to zero at $82 \mathrm{~K}$.

resistance dropping to zero at $82 \mathrm{~K}$. Above $T_{c}$ the resistance is a linear function of temperature which is a characteristic feature of the oxide superconductors. The XRD spectra of these specimens conform to an orthorhomic phase. The typical spectra for the slow-cooled $\mathrm{Y}_{123}$ specimen is shown as Curve [A] in figure 4. The oxygen content of this specimen as determined by the gas chromatographic technique is 6.914 . The TEP of the $\mathrm{Y}_{123}$ specimen (figure 1) is positive, presents a broad maximum around $120 \mathrm{~K}$ and drops to zero value at $T_{c}$. At higher temperature beyond the TEP maximum it is a slow decreasing function of temperature.

The positive sign of TEP in these specimens indicates that the conduction in these materials is via holes. This fact is supported by Hall coefficient measurements (Wang and Ong 1988) and in conformity with most publications on TEP (Srinivasan et al 1987; Crommie et al 1988; Mitra et al 1987; Kang et al 1989; Sera et al 1988; Trodahl and Mawdsley 1987; Ma et al 1989). The peak above $T_{c}$ is a peculiar feature of the TEP of these superconductors observed both in bulk and single-crystal specimens. The peak has been attributed to an enhanced phonon drag effect by Ma et al (1989), Trodahl and Mawdsley (1988) and Uher and Kaiser (1987) which is truncated by the onset of superconductivity. The anomaly was attributed by us (Jha et al 1989a) to pair fluctuation effect. More recently Kumar (1989) modified the free-electron 


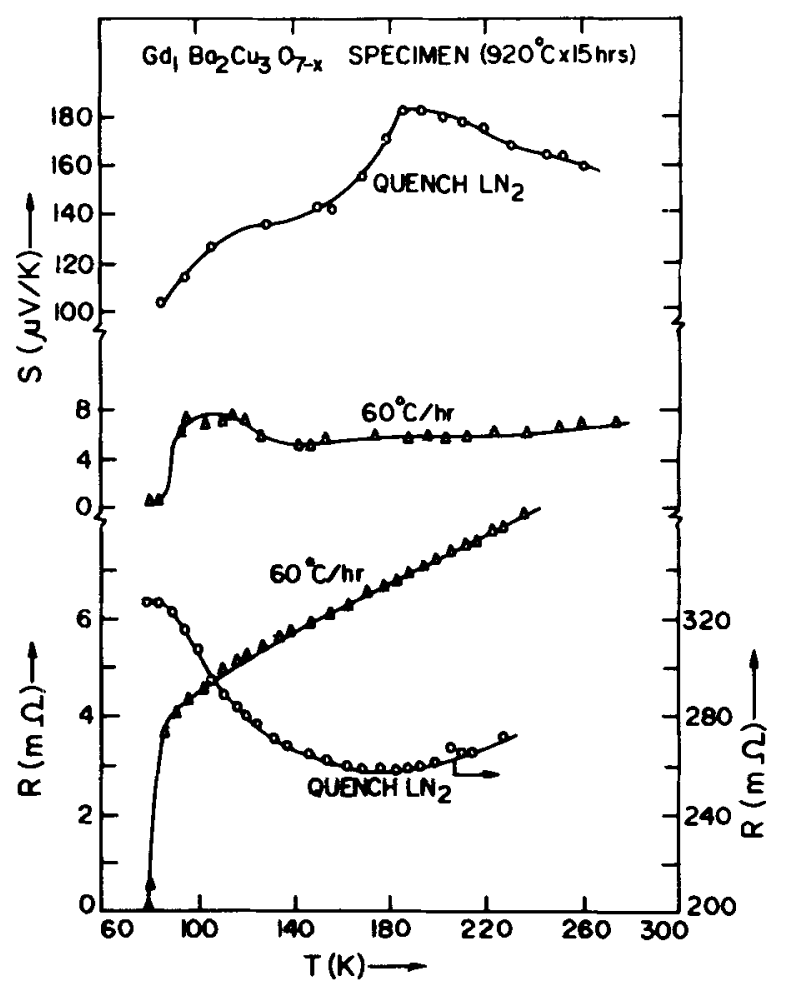

Figure 2. Measured thermoelectric power and electrical resistance of the $\mathrm{Gd}_{1} \mathrm{Ba}_{2} \mathrm{Cu}_{3} \mathrm{O}_{7-x}$ specimens.

expression for TEP given by (MacDonald 1962)

$$
S=\frac{\pi^{2} k_{B}^{2} T}{3 e E_{F}}\left[\frac{\partial \ln \rho(E)}{\partial \ln E}+\frac{\partial \ln v^{2}(E)}{\partial \ln E}+\frac{\partial \ln \tau(E)}{\partial \ln E}\right]_{E=E_{F}}
$$

by taking into account the strong temperature dependence of $\rho(E)$ and $v(E)$ due to pair fluctuation in the normal state. After considering two peculiar features of these oxide superconductors viz. the short coherence length $\xi$ and the 2-D nature, the modified expression given by Kumar (1989) is

$$
S=\frac{\pi^{2} k_{B}}{3 e}\left(\frac{T}{T_{f}}\right)=\frac{1}{1+T_{\xi}|\ln \mu| / 4 T}\left[\frac{1+\left(T_{\xi} T_{f} / 2 T^{2}\right)|\ln \mu|}{1+T_{\xi}|\ln \mu| / 4 T}\right],
$$

where $k_{B} T_{f}=E_{F}, k_{B} T_{\xi}=\hbar^{2} /\left(2 m \xi^{2}\right)$ and $\mu=\left(1-T_{c} / T\right)$. The above expression does explain the anomalous temperature variation of the TEP but gives an exaggerated value of the peak TEP. The effect of disorder, phonon drag or a possible magnon drag (Kang et al 1989) on TEP has probably to be considered.

This typical behaviour of TEP is also found by us in $\mathrm{Gd}_{123}$ (figure 2) and $\mathrm{Sm}_{123}$ (figure 3) systems. The TEP in the $\mathrm{Gd}_{123}$ system at higher temperature is weakly temperature-dependent which is similar to $\mathrm{Y}_{123}$ specimens cooled at faster rates $\left(100-200^{\circ} \mathrm{C} / \mathrm{h}\right)(\mathrm{Jha}$ et al $1989 \mathrm{a})$ with low oxygen stoichiometry $(\sim 6.7)$ and similar 


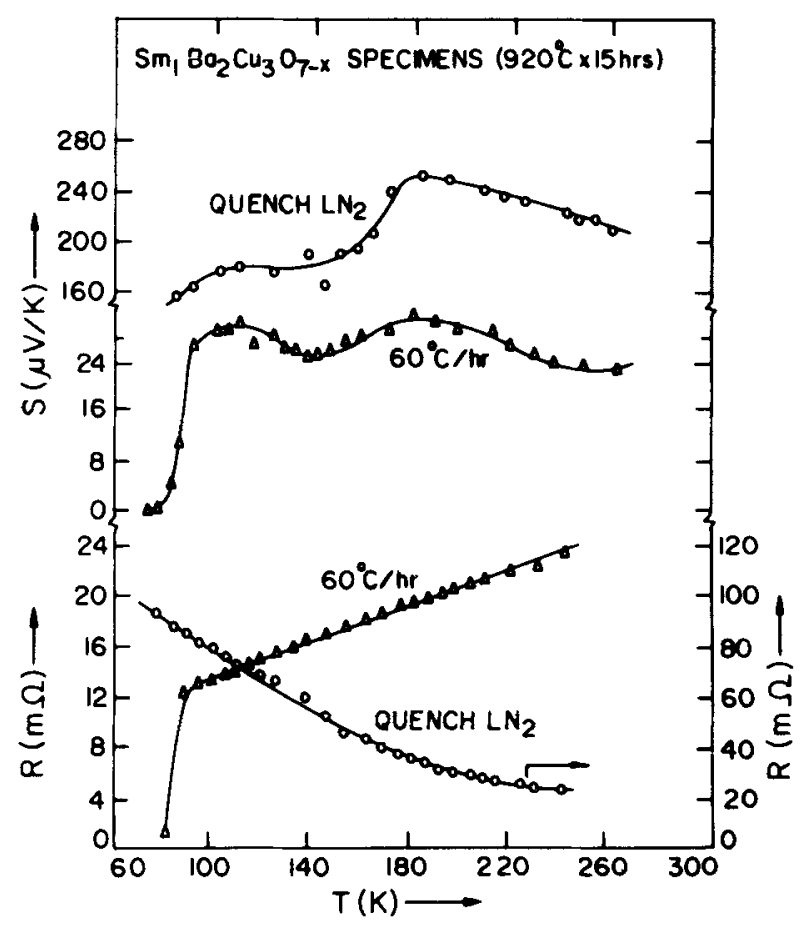

Figure 3. Measured thermoelectric power and electrical resistance of the $\mathrm{Sm}_{1} \mathrm{Ba}_{2} \mathrm{Cu}_{3} \mathrm{O}_{7-x}$ specimens.

to the TEP behaviour observed by Kang et al (1989) for $\mathrm{O}_{2}$-deficient specimen. The $\mathrm{Sm}_{123}$ specimen shows another peak at higher temperature around $180 \mathrm{~K}$ which is quite similar to the peak observed by Trodahl and Mawdsley (1987) for the $Y_{123}$ and $\mathrm{Gd}_{123}$ specimens. This peak has been attributed by them as due to the presence of a small concentration of a semiconductor phase. It thus appears that the peak above $T_{c}$ in TEP in bulk as well as in single crystal (Crommie et al 1988) and epitaxial films (Ma et al 1989) is a special feature of all the $\mathrm{Y}_{123}$ oxide superconductors, the normal state value and temperature variation probably depends strongly on the sample homogeneity and hence on the preparation conditions.

\subsection{Quenched specimens}

Figures 1-3 also show the resistivity and TEP vs temperature plots of the $\mathrm{Y}_{123}, \mathrm{Gd}_{123}$ and $\mathrm{Sm}_{123}$ specimens quenched from the sintering temperature, $920^{\circ} \mathrm{C}$ to $77 \mathrm{~K}$. The $\mathrm{Y}_{123}$ specimen shows a metallic conductivity (figure 1 ) between 300 and $77 \mathrm{~K}$ but does not show a superconducting transition down to $77 \mathrm{~K}$. The specimen, however, shows a resistivity drop below $60 \mathrm{~K}$ followed by a minimum at $45 \mathrm{~K}$ below which resistivity rises again with the decrease of temperature. The $\mathrm{Gd}_{123}$ and $\mathrm{Sm}_{123}$ specimens, on the other hand, show a semiconducting behaviour in resistivity down to $10 \mathrm{~K}$. The X-ray diffraction spectra of the $\mathrm{Gd}_{123}$ and $\mathrm{Sm}_{123}$ specimens show a perfect tetragonal structure which conforms to a semiconducting phase as supported by the 


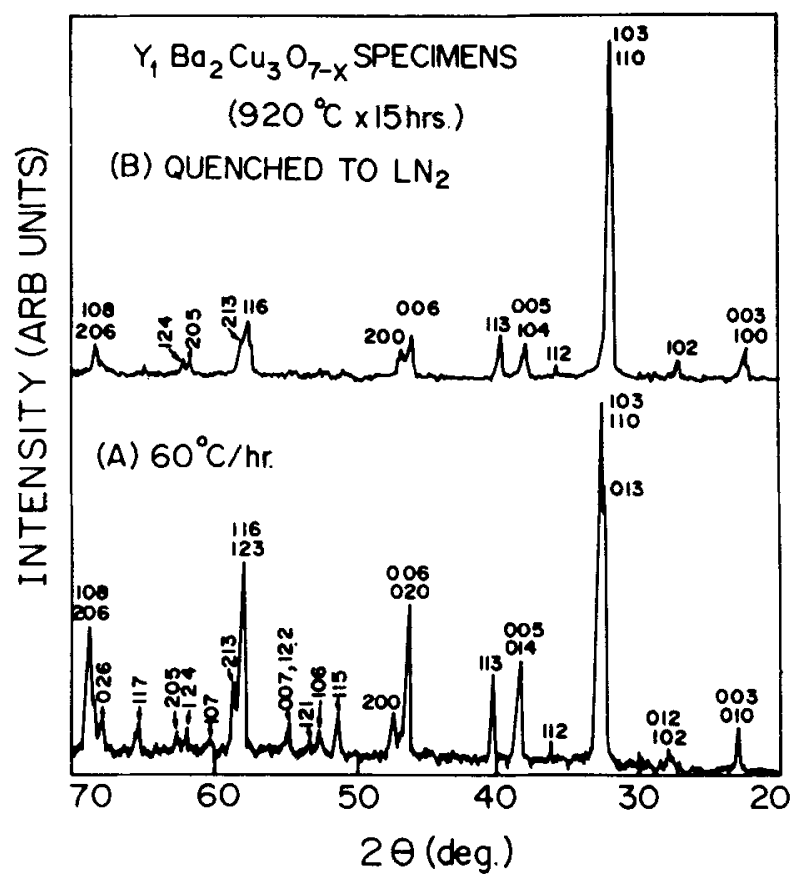

Figure 4. Room temperature $\mathrm{X}$-ray diffraction spectra of $\mathrm{Y}_{1} \mathrm{Ba}_{2} \mathrm{Cu}_{3} \mathrm{O}_{7-x}$ specimens.

respective resistivity data. Figure 4 shows the room temperature XRD spectra of the $\mathrm{Y}_{123}$ specimens slow cooled $60^{\circ} \mathrm{C} / \mathrm{h}$ [A] and quenched to $77 \mathrm{~K}$ [B]. The room temperature XRD spectra [A] conforms to a perfect orthorhombic phase whereas spectra [B] though, appears tetragonal, yet a small amount of an orthorhombic phase can be present. The spectra [B] show all the peaks with different $h$ and $k$ values quite broad which can show splitting in transformation from tetragonal to orthorhombic unit cell. The peaks with the same $h$ and $k$ values like (113) and (112) etc are quite sharp. The quenched $\mathrm{Y}_{123}$ specimen is thus apparently tetragonal, as confirmed by indexing of different reflections up to $2 \theta=80^{\circ}$, with a small amount of orthorhombic distortion $(b-a)$. This X-ray data perfectly agree with the net metallic resistivity, observed in this specimen and a consequent drop in resistivity below $60 \mathrm{~K}$. The resistivity does not drop to zero as there is no continuous superconducting path.

The TEP of all the three quenched specimens is positive, large with peak values $120-250 \mu \mathrm{V} / \mathrm{K}$ appearing between $160 \mathrm{~K}$ and $180 \mathrm{~K}$. All these features correspond to typical semiconductors. As mentioned earlier, with increasing deficiency of oxygen, the hole concentration goes down in the conduction band and the TEP increases. The increase in TEP value of the $Y_{123}$ specimen, is, however, smaller compared to the corresponding increase for $\mathrm{Gd}_{123}$ and $\mathrm{Sm}_{123}$ specimens indicating the presence of at least a small fraction of the orthorhombic phase in this specimen as was also shown by the XRD studies. The most striking feature of the TEP curve for $Y_{123}$ specimen (figure 1) is, however, that it drops to zero around $82 \mathrm{~K}$ which is the $T_{c 0}$ of the slow-cooled $Y_{123}$ specimen. At first sight the resistivity and TEP behaviour of the $Y_{123}$ quenched specimen appears to be incongruent; we, however, emphasize that such a behaviour is possible in such granular superconductors. 


\subsection{Percolation in TEP}

As mentioned earlier these superconductors are granular in nature and the conduction between the grains is via the Josephson coupling. In a composite system such as in situ superconductors (Reddy et al 1986) wherein the superconducting $\mathrm{Nb}$ is dispersed in a normal metal $\mathrm{Cu}$, the composite will be superconducting either when the superconducting volume fraction (SVF) is above the percolation threshold $(\sim 17 \mathrm{~V} \%$ ) (Zallen and Scher 1971) or if the interparticle separation is smaller or comparable to the coherence length. In the latter situation the Cooper pairs can tunnel through from one superconducting particle to another via the proximity effect and superconductivity will be observed. The proximity effect is however suppressed by temperature, current and magnetic field. The well-known drastic decrease of $J_{c}$ with magnetic field at $77 \mathrm{~K}$ in $\mathrm{Y}_{123}$ superconductors (for example Yamada et al 1987) is a clear evidence of the Josephson-coupled conduction in these materials. The Josephson coupling in these materials is very weak, the coherence length being very small 10-20 $\AA$ (Cava et al 1987). At lower temperatures the Josephson coupling increases and $J_{c}$-magnetic field variation is less drastic.

In the present studies the quenched $\mathrm{Y}_{123}$ specimen is considered to be a composite consisting of a tetragonal matrix with a dispersion of orthorhombic superconducting grains. The XRD studies clearly show that the orthorhombic phase present is close to its detection limit $(\sim 5 \%)$, the percolation path as envisaged for electrical conduction mechanism is ruled out. The other mechanism for conduction just discussed viz the proximity effect also does not appear to operate, the coherence length being only $10-20 \AA$.

A metallic but finite resistivity of this specimen down to $77 \mathrm{~K}$ supports these arguments. The drop in resistivity below $60 \mathrm{~K}$ and a minimum at $45 \mathrm{~K}$ could be due

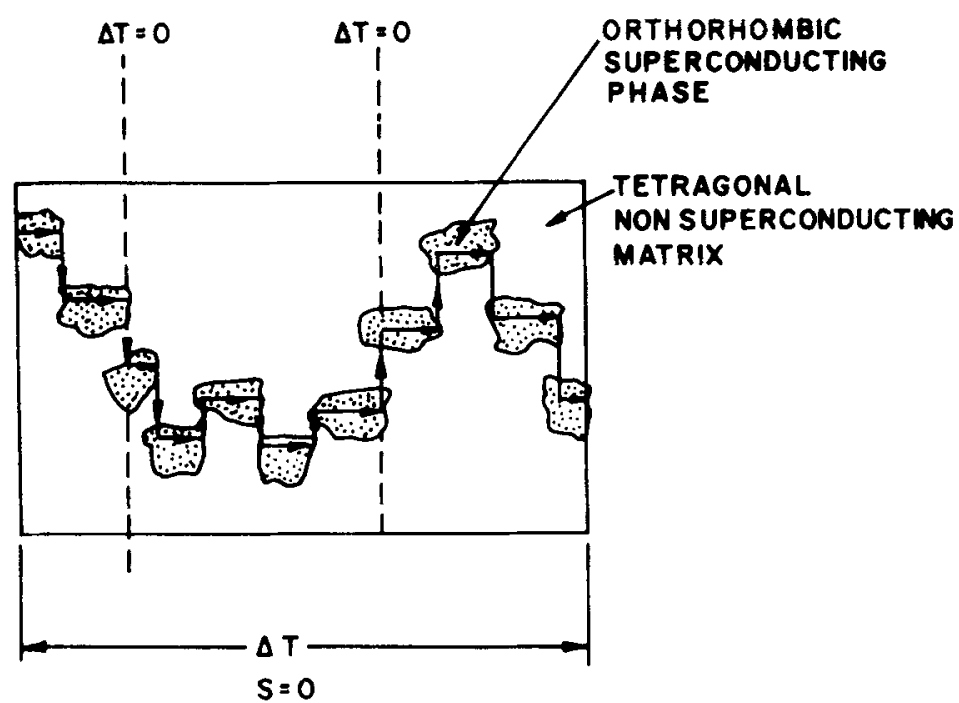

Figure 5. A percolation mechanism for zero thermoelectrical power in a composite consisting of superconducting grains in a non-superconducting matrix. The grains widely separated in transverse direction do have overlapping along the longitudinal direction. 
to the increase in Josephson coupling with the fall of temperature. The semiconducting phase, however, ultimately takes over the specimen below $45 \mathrm{~K}$.

The TEP of this specimen surprisingly drops to zero at $82 \mathrm{~K}$, the transition temperature of the $60^{\circ} \mathrm{C} / \mathrm{h}$ cooled $Y_{123}$ specimen. Visualizing the specimen (figure 5) as consisting of isolated superconducting grains in a tetragonal matrix (Jha et al $1989 \mathrm{~b}$ ) it is easy to see that the sample would have a finite resistivity as the charge carriers have to cross the normal matrix transversely from one grain to another. In TEP measurement a temperature gradient is applied along the longitudinal direction as shown in figure 5. A charge carrier will move under the influence of $\Delta T$ longitudinally along the superconducting grain without developing a thermo-emf. Similarly there will be no thermo-emf when a charge carrier crosses the matrix from one grain to another in a transverse direction, $\Delta T$ along any transverse cross-section being zero. Such a situation will be valid so long as there is overlapping of superconducting grains along the longitudinal direction irrespective of their separation along the transverse direction. It thus appears that the critical concentration of a superconducting phase required to make the TEP of the composite zero is much smaller than that required for the resistivity to be zero. An analytical estimate supported by numerical simulations has been made by Rajput and Kumar (1989) for the percolation threshold for which zero TEP paths would exist similar to those shown in figure 5 . The basis of their calculations is that such paths would exist, if the projection of the occupied (superconducting) fraction on a line parallel to the temperature gradient percolates. For 3-dimensions, their calculations show that such a threshold concentration would be $6 \%$ which is a reasonable value and close to the value estimated by us from our experimental data.

To test this prediction of a very small critical threshold for zero TEP in composite superconductors we are conducting experiments on multiphase BSCCO system as well as on specimens prepared from the mixtures of superconducting and non-superconducting phases of the $\mathrm{Y}_{123}$ systems. We do find similar experimental evidence. The TEP in many specimens drops to zero at the transition temperature even though the resistivity is either finite or does not even show a transition.

\section{Conclusions}

The thermoelectric power of the $\mathrm{R}_{1} \mathrm{Ba}_{2} \mathrm{Cu}_{3} \mathrm{O}_{7-x}$ compounds $(\mathrm{R}=\mathrm{Y}, \mathrm{Gd}$ and $\mathrm{Sm})$ between 300 and $77 \mathrm{~K}$ is positive, exhibits a peak above $T_{c}$ and decreases with increase of temperature. Pair fluctuation is thought to be the possible reason for this anomalous behaviour. Highly $\mathrm{O}_{2}$-deficient $\mathrm{Y}_{123}$ specimen shows zero TEP at $T_{c}$ even though it has a finite resistivity. It is concluded that the critical percolation concentration for TEP in a composite material is far smaller than the percolation threshold for electrical conduction.

\section{Acknowledgements}

We would like to thank Prof. S K Joshi for his keen interest in this work. We also thank Prof. D Kumar of the Jawaharlal Nehru University for many stimulating 
discussions and for having shown interest in carrying out analytical calculations. One of us (SRJ) would like to thank Prof. R Nath (Delhi University) and the UGC for a senior fellowship.

\section{References}

Cava R J et al 1987 Phys. Rev. Lett. 581676

Crommie M F, Zettl A, Barbee III T W and Cohen M L 1988 Phys. Rev. B37 9734

Deutscher G, Entin-Wohlman O, Fishman S and Shapira Y 1980 Phys. Rev. B21 5041

Dinger T R, Worthington T K, Gallagher W J and Sandstorm R L 1987 Phys. Rev. Lett. 582687

Hagen S, Jing T W, Wang Z Z, Horwath J and Ong N P 1988 Phys. Rev. B37 7928

Jha S R, Reddy Y S, Suri D K, Kundra K D, Sharma R G and Kumar D 1989a Pramana-J. Phys. 32277

Jha S R, Reddy Y S and Sharma R G 1989b Pramana-J. Phys. 33 L615

Jorgensen J D et al 1987 Phys. Rev. B36 3608

Kang W N, Cho K C, Kim Y M and Choi Mu-Yong 1989 Phys. Rev. B39 2763

Kumar D 1989 Preprint

Ma H, Xiong G, Wang L, Wang S, Zhang H, Tong L, Liang S and Yan S 1989 Phys. Rev. B40 9374

Martin S, Fiory T, Fleming R M, Schneemeyer L F and Waszczak J V 1988 Phys. Rev, Lett. 602194

Mitra N, Trefny J, Young M and Yarar B 1987 Phys. Rev. B36 5581

Parashar D C, Rai J, Gupta P K, Sharma R C and Lal K 1988 Jpn. J. Appl. Phys. 27 L2304

Rajput R and Kumar D 1989 Proceedings Int. Conf. on Superconductivity, Bangalore, India; 1990 Phys. Rev. B42 8634

Reddy Y S, Krishna M M and Sharma R G 1986 J. Low Temp. Phys. 65261

Sera M, Shamoto S and Sato M 1988 Solid State Commun. 68649

Sharma R G, Reddy Y S, Jha S R and Dubey S S 1988 Pramana-J. Phys. 30 L81

Srinivasan R, Shankarnarayana V, Raju N P, Natarajan S, Varadaraju U V and Subbarao G V 1987 Pramana-J. Phys. 29 L.225

Taylor K N R, Shepherd M, Mathews D N, Russel G J, Bailey A D and Sealy K 1988 in High temperature superconductors II (eds) D W Capone II, W H Butler, B Batlogg and C W Chu (Nevada: MRS Publication) p. 247

Trodahl H J and Mawdsley A 1987 Phys. Rev. B36 8881

Wang Z Z and Ong N P 1988 Phys. Rev. B38 7160

Yamada Y et al 1987 Jpn J. Appl. Phys. 261205

Zallen R and Scher H 1971 Phys. Rev. B4 4471 